\title{
A Comparative Study Between Different Surgical Techniques For Left Atrial Exclusion in Patients Undergoing Concomitant Cardiac Surgery
}

\author{
Ahmed Abdeljawad, MD, ${ }^{1,2}$ Yasser Shaban Mubarak, MD ${ }^{3}$ \\ ${ }^{1}$ Department of Cardiothoracic Surgery, Cairo University, Cairo, Egypt; \\ ${ }^{2}$ Clinic of Cardiac Surgery, Barmherzigen Brüder Krankenhaus Trier, Trier, Germany; \\ ${ }^{3}$ Madinah Cardiac Center, Minia University, Minia, King Fahd Hospital, KSA, Egypt
}

\section{ABSTRACT}

Objectives: To find out the most successful surgical technique to obliterate left atrial appendage (LAA) in atrial fibrillation $(\mathrm{AF})$ patients who had undergone concomitant cardiac surgery.

Background: About $10 \%-65 \%$ of patients develop AF following cardiac surgery [Rho 2009; Mathew 2004; Maesen 2012]. Cerebral cardio-embolic stroke remains the most serious complication in AF patients. LAA is the main anatomical source for thromboembolic events. The use of oral anticoagulants (OAG) is considered to be an effective method for reduction of thromboembolic complications [Johnson 2000]. The use of oral anticoagulants is faced by two important facts which are the therapy duration is still unknown [Kirchhof 2017] and importantly that between $30-50 \%$ of patients are not candidates for oral anticoagulants due to the high bleeding risk or other contraindications [Johnson 2000; Kirchhof 2017; Kirchhof 2014]. In such patients, LAA obliteration would be an optimal alternative technique as it will reduce the stroke risk by $50 \%$ [Go 2014]. Several surgical techniques with variable degrees of success rates have been used. It still is unclear which surgical technique is optimum to achieve a successful obliteration of the LAA and a considerable reduction of the postoperative stroke events in $\mathrm{AF}$ patients.

Patients and methods: A total of 100 patients have been subjected to surgical LAA exclusion from April 2017 to April 2019 in two different centers. All patients had postoperative transesophageal echo (TEE) examination to confirm the success of LAA occlusion. All patients included in our study suffered from $\mathrm{AF}$ at the time of surgery or in past history, which was confirmed by ECG examination in their previous medical files. A variety of surgical techniques to close the LAA have been utilized, including surgical excision by means of scissors, patch exclusion by means of an endocardial patch, suture exclusion and finally stapler exclusion. TEE examination 16

Received December 6, 2020; received in revised form April 15, 2021; accepted April 16, 2021.

Correspondence: Abmed Abdeljawad, Telephone +491638144990;

+201221152534. (e-mail: ahmed.gawad@kasralainy.edu.eg). months postoperatively divided our patients into four groups as follows: successful LAA occlusion, Patent LAA, excluded LAA with persistent flow into LAA, and remnant LAA with a stump connection with LAA more than $1 \mathrm{~cm}$.

Results: Out of 100 patients, 30 patients (30\%) underwent surgical LAA excision, 24 patients $(24 \%)$ underwent surgical epicardial suture ligation, eight patients $(8 \%)$ underwent patch exclusion using autologous pericardial patch, 33 patients $(33 \%)$ underwent LAA internal orifice purse string suture obliteration, and five patients $(5 \%)$ underwent stapler exclusion. Forty-two patients out of 100 (42\%) showed successful LAA closure. The successful LAA occlusion occurred mostly in LAA excision patients $87 \%, 24 \%$ in LAA internal orifice purse string suture obliteration patients, $21 \%$ in epicardial suture ligation patients, and $37.5 \%$ in patch exclusion patients. The stapler exclusion was very disappointing as we did not record a single case out of the five patients who showed a successful LAA occlusion. Stroke events were recorded in all surgical techniques except the LAA excision technique. The stroke rate after two years follow up was zero in the surgical excision group, $49 \%$ in the suture exclusion group, $20 \%$ in the patch exclusion group, and $40 \%$ in stapler exclusion group.

Conclusion: Surgical LAA excision is the most successful technique for LAA occlusion and represents a promising technique for the reduction of thromboembolic events in $\mathrm{AF}$ patients who undergo a concomitant cardiac surgery.

\section{INTRODUCTION}

Atrial fibrillation is the most common cardiac rhythm disorder of clinically significant value [Go 2014]. It is one of the significant etiologies of cardiac ischemic strokes, increasing stroke probability five times in comparison to patients who do not suffer from atrial fibrillation [Wolf 1991]. The annual stroke risk in $\mathrm{AF}$ patients reaches about $5 \%$ and increases with concomitant risk factors such as age, cardiac valvular diseases, hypertension, and left ventricular dysfunction [No authors listed 1994; Benjamin 1994]. Up to $90 \%$ of left atrial clots originate in the left atrial appendage in case of non-rheumatic AF [Blackshear 1996; Manning 1994].

Anticoagulation therapy either with vitamin-K antagonists or with recent direct oral anticoagulants which directly inhibit factor $\mathrm{Xa}$ are still considered to be the first line treatment 
to reduce thrombus formation within LAA [Kirchhof 2016]. However, anticoagulation therapy still leads to a high risk of bleeding in some high bleeding risk patients, such as elderly patients with hepatic or renal dysfunction, and increased risk of cerebral bleeding in such patients [Friberg 2015; Del-Carpio 2016].

Based on previous drawbacks of the pharmacological treatment the surgeons started to search for a non-pharmacological approach to minimize the risk of thrombus fragmentation and migration from LAA. The first surgical LAA excision was made in 1949 by Madden, who had resected LAA during mitral valve surgery in patients who had thrombi inside them [Madden 2018]. This procedure gained a little attention until late 1980s and early 1990s, when Cox et al. introduced a surgical procedure to treat AF, which was known as Cox-Maze III, when the authors noticed in their long-term follow-up study that the low rate cerebral embolic strokes in their patients was not only attributed to successful sinus rhythm restoration but also attributed to eliminating risk of thrombi in their LAA [Cox 1999].

Surgical LAA occlusion can be performed by excision using surgical scissors, staplers, LAA suture exclusion via epicardial suture ligation, patch exclusion using autologous pericardial patch, LAA internal orifice obliteration using $4 / 0$ Polypropylene purse string double suture or the placement of an occlusion device, such as atriclip.

Surgical excision of LAA has been shown in several trials to be better than other occlusion methods regarding thrombus formation and cerebrovascular stroke events [Lee 2013]. However, the risk of bleeding in surgical excision of LAA is more than other occlusion methods, especially in patients with fragile left atrial tissues or redo patients [Kanderian 2008]. Such patients should be considered candidates for other occlusion techniques, such as internal ligation or external occlusion.

Surgical LAA exclusion - preferably via surgical appendage excision - was recommended in patients at high risk of LAA associated thromboembolism who are undergoing a concomitant cardiac surgery, underwent a previously failed percutaneous LAA exclusion, or who will undergo an isolated LAA exclusion procedure [Greenberg 2019].

Because the majority of surgical LAA closure studies were designed retrospectively and mostly performed at a single center, we performed our study prospectively and in two different centers to find out which surgical technique would better reduce the risk of thromboembolic stroke events.

\section{PATIENTS AND METHODS}

A total of 100 patients were subjected to surgical LAA exclusion, from April 2017 to April 2019, in two different cardiac surgery centers in a prospective randomized cohort study. Each patient confirmed they read and understood the information about the research, as provided in the participant information sheet within their file. The study got formal approval and permission from the heart team meeting and cardiac surgery departments before starting the study. The study conformed to the principles of the "Declaration of Helsinki," and the investigator followed the appropriate safeguards regarding the rights and welfare of the human participants included in the study.

Surgical techniques included surgical LAA excision using surgical scissors, LAA suture exclusion via epicardial suture ligation, patch exclusion using autologous pericardial patch, LAA internal orifice obliteration using 4/0 Polypropylene purse string double suture placed around the base of the LAA from the internal aspect of LAA and finally stapler exclusion. Our inclusion criteria included older patients with an age $\geq 56$ years, past or present history of $\mathrm{AF}$, and first-time open heart surgery (isolated mitral valve surgery or a combined mitral valve surgery and $\mathrm{CABG}$ ). Our exclusion criteria included redo surgery, off pump, double valve operations and cardiogenic shock.

We preferred to include mitral valve surgery or a combined mitral valve surgery and CABG, as all patients were rheumatic even associated with coronary artery diseases (CAD). There was no preoperative preference for the technique of the LAA occlusion that would be used intraoperatively to occlude the LAA. The decision to choose one of the previously mentioned techniques was taken intraoperatively, depending on the size of the LAA, degree of the fragility of the LAA tissues, and the feasibility to ligate or excise the LAA which depended on its depth in relation to the mediastinum.

Regarding our surgical techniques, we used scissors to excise the left atrial appendage. (Figure 1) Epicardial suture exclusion was performed by means of an epicardial ligation using number 1 silk ligature. For LAA internal orifice obliteration using 4/0 Polypropylene purse string double suture placed around the base of the LAA, the stitches should encircle the oval shaped internal orifice of the LAA or obliterate LAA orifice in two layers transverse mattress and continuous. (Figure 2) Stapler exclusion was performed using a reloadable linear stapler. Patch exclusion was done by means of a nontreated autologous pericardial patch using 4-0 polypropylene running suture technique.

There are several surgical techniques used to obliterate the LAA, and TEE was chosen to determine which surgical technique was the most successful to achieve permanent closure of LAA after a follow-up period of 16 months. LAA thrombi were detected as dense smoke-like echoes or spontaneous echo contrast (SEC) in the left atria.

In order to assess the presence or absence of the flow between the LA and LAA, color Doppler was performed. Accordingly, we classified the LAA echocardiographically into four groups:

1. Successful closure, which is considered the only successful end result.

2. Excluded LAA with persistent flow between the left atrium and the LAA that needed to be obliterated.

3. Remnant LAA, which is defined as a residual pouch or stump communicating between the LA and the LAA more than $1 \mathrm{~cm}$.

4. Totally patent LAA with a free flow between LA and LAA. 


\section{RESULTS}

A total of 100 patients were included in our study. The mean age was 60 years. Male gender was present in $68 \%$ of LAA surgical excision patients, $43 \%$ in LAA internal orifice purse string suture obliteration, $62 \%$ of the LAA surgical exclusion group, and $60 \%$ of LAA stapler exclusion. (Table 1)

Patients who had AF at the time of presentation were $60 \%$ of LAA surgical excision, $66 \%$ of the LAA internal orifice purse string suture obliteration, $78 \%$ of the LAA surgical exclusion, and $60 \%$ of the LAA stapler exclusion.

Risk factors were similar in all groups, except stapler exclusion, because of the small number of patients. There was no statistically significant difference in the preoperative patient characteristics or risk factors with a $P$-value ranging between 0.3 and 0.9 . In case of isolated mitral valve surgery, all techniques were performed, however combined $\mathrm{CABG}$ and mitral valve surgery, purse-string ligation were the most common technique. (Table 2)

After 16 months follow up for the results of the different surgical techniques to obliterate the LAA we obtained the following results:

1. Among the 30 patients who underwent surgical excision of LAA and after 16 months follow up, there were no cases of patent LAA or persistent flow. Only four patients (13\%) were found to have remnant LAA with a stump or a pouch more than $1 \mathrm{~cm}$. The success rate of complete LAA closure was $87 \%$, which was statistically significant with a $P$-value $<0.002$.

2. Among the 24 patients who underwent LAA suture exclusion, $46 \%$ of these patients were found to have excluded LAA with a persistent flow. Totally patent LAA and a remnant LAA with a stump more than $1 \mathrm{~cm}$ were found in a similar incidence $16.6 \%$. Successful LAA closure was present in only one-fifth of these patients (21\%).

3. Among the eight patients who underwent an endocardial patch exclusion of LAA, three patients $(37.5 \%)$ proved to have a successful LAA closure.

4. Among the 33 patients who underwent LAA internal orifice purse string suture obliteration, eight patients (24\%) proved to have a successful LAA closure.

5. Among the five patients who underwent stapler LAA exclusion, no patients were found to get a successful closure of their LAA.

The key finding in our study was that 52 out of 100 patients $(52 \%)$ had a successful LAA closure. Successful LAA closure has been documented to be more with LAA excision (87\%), which was statistically significant with a $P$-value $<0.002$ vs. patch exclusion $(37.5 \%)$ vs. LAA internal orifice purse string suture obliteration (24\%) vs. suture exclusion (20\%) vs. stapler exclusion where we did not find a single patient with a successfully closed LAA after 16 months of follow up. (Table 3)

Postoperative stroke or TIAs events after different surgical techniques:

- No patient who underwent LAA excision had experienced stroke or TIAS.

- Seven patients (21\%) of those who underwent LAA internal orifice purse string suture obliteration developed a

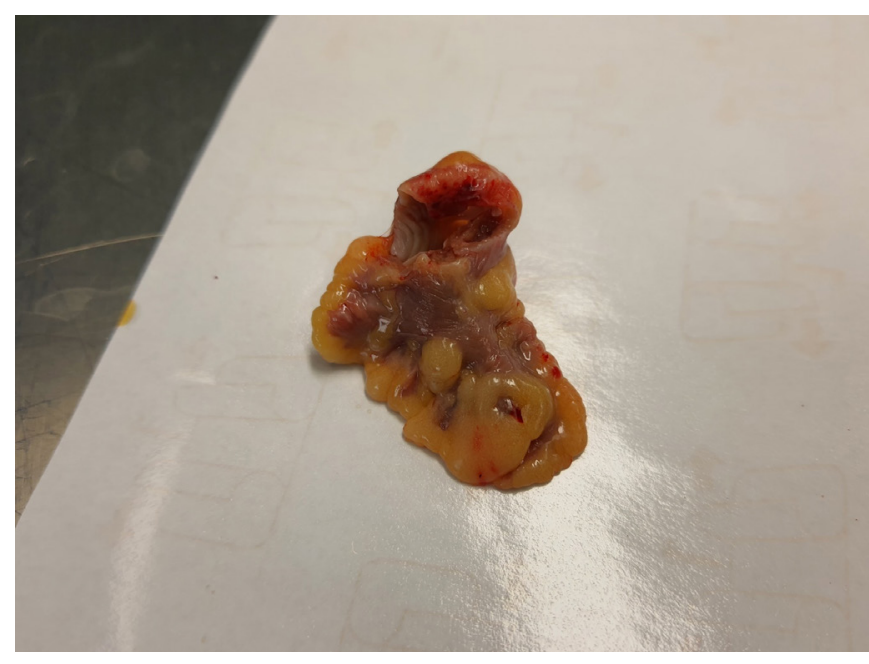

Figure 1. Excised left atrial appendage

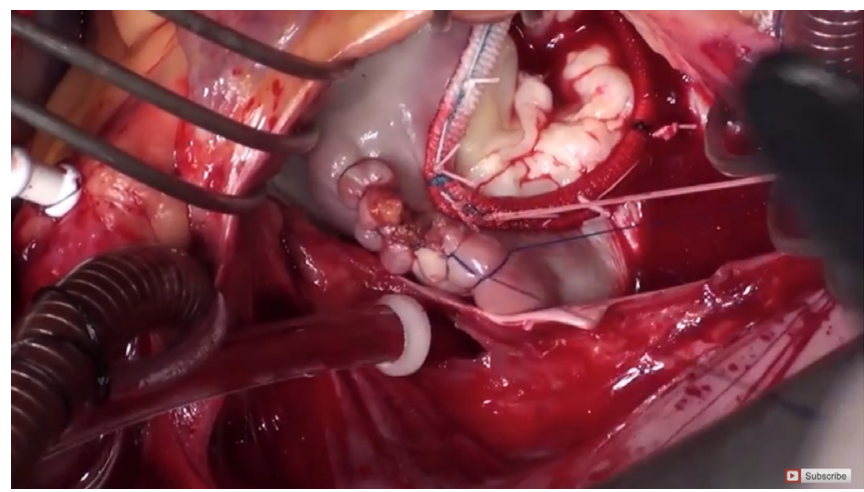

Figure 2. Obliterated LAA orifice using two layers polypropylene transverse mattress and continuous

stroke or TIAs. Four of these (12\%) occurred after oneyear follow up, and three patients (9\%) developed this after two-years follow up.

- Seven patients (28\%) of those who underwent LAA external suture exclusion developed a stroke or TIAs. This happened with three patients (12\%) after one-year follow up and four patients (16\%) after two-years follow up.

- Only one patient (12.5\%) of eight patients who underwent a patch exclusion of LAA developed TIAs event after two years.

- Two patients (40\%) of the LAA stapler exclusion patients developed a stroke event after one year.

It is worthwhile to note that there was no postoperative bleeding necessitating a re-exploration operation. All thromboembolic events encountered in our study were recorded under complete pharmacological anticoagulation coverage.

It is also worthwhile to mention that bipolar/monopolar radiofrequency atrial ablation using the cobra device to induce the box lesion was performed in 14 patients (10 patients from the LAA internal orifice purse string suture obliteration group and four patients from the LAA excision group). All 
patients included in our study were discharged from the hospitals without introducing any modifications on the preoperative anticoagulation regimen that was prescribed for them as a prophylaxis against thromboembolic events.

\section{DISCUSSION}

The majority of Surgical LAA exclusion studies are carried out retrospectively and mostly performed at a single center. However, our study is prospective in design and was performed in two different centers.

Recently, there has been an enormous trend in occlusion of LAA by various surgical and non-surgical approaches and techniques. The surgical LAA exclusion techniques include surgical LAA excision using surgical scissors, suture exclusion via epicardial suture ligation or endocardial purse-string suture of internal orifice occlusion and patch exclusion using autologous pericardial patch.

In a study performed by Lee et al. in 2013, a study of 710 patients who underwent concomitant AF surgery, reported that only 25 patients out of $710(3.5 \%)$ had developed stroke or TIAs in the postoperative period. The rate of stroke or TIAs was lower in the group of patients who had received LAA exclusion by means of surgical excision as compared with those who had received LAA exclusion by other surgical exclusion techniques [Lee 2013]. The incidence of postoperative stroke \pm TIA in our study was (24\%) after one year of follow up in

Table 1. Characteristics of patients undergoing LAA closure by various surgical techniques

\begin{tabular}{|c|c|c|c|c|}
\hline Variables & $\begin{array}{l}\text { Surgical excision } \\
\qquad(N=30)\end{array}$ & $\begin{array}{l}\text { Purse-string suture oblitera- } \\
\text { tion } \\
(N=33)\end{array}$ & $\begin{array}{l}\text { Surgical exclusion (epicardial } \\
\text { ligation and patch exclusion) } \\
\qquad(N=32)\end{array}$ & $\begin{array}{l}\text { Stapler exclusion } \\
\qquad(N=5)\end{array}$ \\
\hline Age & $60 \pm 4$ years & $58 \pm 2$ years & $62 \pm 6$ years & $61 \pm 5$ years \\
\hline Gender (male) & $17(56 \%)$ & $14(43 \%)$ & $20(62 \%)$ & $3(60 \%)$ \\
\hline $\mathrm{AF}$ at the time of presentation & $18(60 \%)$ & $22(66 \%)$ & $25(78 \%)$ & $3(60 \%)$ \\
\hline Hypertension & $16(53 \%)$ & $18(54 \%)$ & $20(62 \%)$ & $3(60 \%)$ \\
\hline DM & $12(40 \%)$ & $14(42 \%)$ & $14(43 \%)$ & $2(40 \%)$ \\
\hline Hyperlipidemia & $15(50 \%)$ & $18(54 \%)$ & $13(40 \%)$ & $2(40 \%)$ \\
\hline Left atrial size & $4.7 \pm 1.2$ & $4.6 \pm 1.1$ & $4.8 \pm 0.9$ & $4.5 \pm 0.6$ \\
\hline Mitral valve surgery & $22(73.3 \%)$ & $20(60.6 \%)$ & $26(81.2 \%)$ & $3(60 \%)$ \\
\hline Combined CABG and mitral valve surgery & $8(26.6 \%)$ & $13(39.3 \%)$ & $6(18.7 \%)$ & $2(40 \%)$ \\
\hline
\end{tabular}

Table 2. Success of different surgical techniques to close LAA after 16 months follow up

\begin{tabular}{lccc}
\hline Variable & Patent LAA & Remnant LAA & Persistent flow LAA \\
\hline Surgical excision (30) & 0 & $4(13 \%)$ & 0 \\
Internal orifice purse-string suture (33) & $5(15 \%)$ & $5(15 \%)$ & $15(46 \%)$ \\
Suture exclusion (epicardial ligation) $(24)$ & $4(16.6 \%)$ & $4(16.6 \%)$ & $11(46 \%)$ \\
Patch exclusion (8) & $1(12.5 \%)$ & $1(12.5 \%)$ & $3(37.5 \%)$ \\
Stapler exclusion (5) & $1(20 \%)$ & $1(20 \%)$ & $3(60 \%)$
\end{tabular}

Table 3. Postoperative stroke or TIAs events after different surgical techniques

\begin{tabular}{lc}
\hline Surgical technique to close the LAA & After one year follow up \\
\hline Surgical excision & 0 \\
Internal suture closure & $4(12 \%)$ \\
External suture closure & $3(12 \%)$ \\
Patch exclusion & 0 \\
Stapler exclusion & $2(40 \%)$
\end{tabular}


both groups of patients who underwent external suture exclusion and LAA internal orifice purse-string suture obliteration, with $N=7$ out of 57 patients and (24\%) after two years of follow up in the same groups of patients who underwent suture exclusion, with $N=7$ out of 57 patients. We reported only one case of stroke (20\%) after two years in the group of patients who underwent pericardial patch LAA orifice obliteration. In our study, we did not report any case of stroke or TIAs after one or two years of follow up in the group of patients who had underwent surgical LAA Excision by scissors.

These results are similar to the results concluded by Kanderian et al. in 2008, that had recorded a total of 18 patients out of 137 patients (13\%) who experienced stroke/TIAs. Six patients out of 18 (4\%) underwent LAA excision and 11 patients out of $18(8 \%)$ underwent surgical exclusion, which lends further credibility to confirmation that surgical excision of LAA is preferable to other surgical exclusion techniques in reducing the risk of postoperative stroke and TIAs.

The results of our study show that LAA successful closure was achieved in 52 patients out of 100 patients (52\%). In reference to the various surgical techniques that we used in our study, we found that LAA excision by scissors to be the most effective with a success rate of $87 \%$ with a $P$-value $<0.002$.

In comparison to a study performed by Kanderian et al. [Kanderian 2008], our LAA management was unsuccessful in 48 patients out of 100 patients (48\%). LAA management was unsuccessful in their study in nearly $60 \%$ of patients. Of the various surgical techniques, excision was the most effective with a success rate of $87 \%$, as compared with a $73 \%$ success rate in the previous study. However, we have to bear in mind that our study was performed on nearly two-thirds of the number of patients (100) as compared with their study with 137 patients.

We noticed in our study that there is a high percentage of LAA suture exclusion patients who developed postoperative persistent flow into LAA (46.5\%) as compared with $61 \%$ in the study performed by Kanderian et al. [Kanderian 2008].

Stapler exclusion was found to be the technique with the highest unsuccessful results in our study with postoperative remnant LAA with a stump more than $1 \mathrm{~cm}$ in $20 \%$ of our patients who underwent stapler exclusion, compared with $58 \%$ in the Kanderian et al. study. The technique of stapler exclusion revealed unsuccessful results with persistent flow into LAA in $60 \%$ of our patients, as compared with $25 \%$ in the same previous study.

\section{REFERENCES}

Benjamin EJ, Levy D, Vaziri SM, D’Agostino RB, Belanger AJ, Wolf PA. 1994. Independent risk factors for atrial fibrillation in a populationbased cohort. The Framingham Heart Study. JAMA. 271:840- 44.

Blackshear JL, Odell JA. 1996. Appendage obliteration to reduce stroke in cardiac surgical patients with atrial fibrillation. Ann Thorac Surg. 61:755-9.

Cox J, Ad N, Palazzo T. 1999. Impact of the Maze procedure on the stroke rate in patients with atrial fibrillation. J Thorac Cardiovasc Surg. 118:833-40.
Del-Carpio Munoz F, Gharacholou SM, Munger TM, Friedman PA, Asirvatham SJ, Packer D1, et al. 2016. Meta-analysis of renal function on the safety and efficacy of novel oral anticoagulants for atrial fibrillation. Am J Cardiol. 117:69-75.

Friberg L, Benson L, Lip GY. 2015. Balancing stroke and bleeding risks in patients with atrial fibrillation and renal failure: the Swedish Atrial Fibrillation Cohort study. Eur Heart J. 36:297-306.

Go AS, Mozaffarian D, Roger VL, Benjamin EJ, Berry JD, Blaha MJ, et al. 2014. Heart disease and stroke statistics-2014 update: a report from the American Heart Association Circulation. 129(3):e28-e292.

Greenberg JW, Lee R, Hui DS. 2019. Patient selection and methods of surgical left atrial appendage exclusion. J Thromb Thrombolysis. 48:209-14.

Johnson WD, Ganjoo AK, Stone CD, Srivyas RC, Howard M. 2000. The left atrial appendage: our most lethal human attachment! Surgical implications. Eur J Cardiothorac Surg. 17(6):718-22.

Kanderian AS, Gillinov AM, Pettersson GB, Blackstone E, Klein AL. 2008. Success of surgical left atrial appendage closure: assessment by transesophageal echocardiography. J Am Coll Cardiol. 52:924-29.

Kirchhof P, et al. 2017. 2016 ESC Guidelines for the Management of Atrial Fibrillation Developed in Collaboration With EACTS. Rev Esp Cardiol (Engl Ed). 70(1):50.

Kirchhof P, Ammentorp B, Darius H, de Caterina R, le Heuzey JY, Schilling RJ, et al. 2014. Management of atrial fibrillation in seven European countries after the publication of the 2010 ESC guidelines on atrial fibrillation: primary results of the prevention of thromboembolic eventsEuropean registry in atrial fibrillation (PREFER in AF). Euro pace. 16(1):6-14.

Kirchhof P, Benussi, Kotecha D, Ahlsson A, Atar D, Casadei B, et al. 2016. ESC guidelines for the management of atrial fibrillation developed in collaboration with EACTS. Euro J Cardiothorac Surg. 50: el-e88.

Lee R, Jivan A, Kruse J, McGee EC, Malaisrie SC, Bernstein R, et al. 2013. Late neurologic events after surgery for atrial fibrillation: rare but relevant. Ann Thorac Surg. 95:126-131.

Madden JL. 2018. Surgical management of the left atrial appendage: a must or a myth? Euro J Cardiothorac Surg. 53: 33-38.

Maesen B, Nijs J, Maessen J, Allessie M, Schotten U. 2012. Postoperative atrial fibrillation: a maze of mechanisms. Europace. 14(2):159-74.

Manning WJ, Silverman DI, Katz SE, Riley MF, Come PC, Doherty RM, et al. 1994. Impaired left atrial mechanical function after cardioversion: relation to the duration of atrial fibrillation. J Am Coll Cardiol. 23:1535-40.

Mathew JP, Fontes ML, Tudor IC, Ramsay J, Duke P, Mazer CD, et al. 2004. A multicenter risk index for atrial fibrillation after cardiac sur- gery. JAMA. 291(14):1720-9.

No authors listed. 1994. Risk factors for stroke and efficacy of antithrombotic therapy in atrial fibrillation. Analysis of pooled data from five randomized controlled trials. Arch Intern Med. 154: 1449-57.

Rho RW. 2009. The management of atrial fibrillation after cardiac surgery. Heart. 95(5):422-9.

Wolf PA, Abbott RD, Kannel WB. 1991. Atrial fibrillation as an independent risk factor for stroke: the Framingham Study: Stroke. 22 (8):983-8. 